\title{
The Navigation Method of Formation Flying Satellites Based on Baseline Information and Pulsars
}

\author{
Kun Zhao, Muqing Li, L.P.Xu*, Jijun Li and Yu Chen \\ ${ }^{1}$ School of Aerospace Science and Technology, XIDIAN university, China \\ ${ }^{*}$ Corresponding author
}

\begin{abstract}
The method of X-ray pulsar-based navigation can be applied to deep space exploration and earth-orbiting spacecraft navigation for its good performance in reliability and autonomy. But high-precision navigation can be hardly achieved when the input of navigation system is merely the signal of pulsars. It infers the navigation system is hardly meet the requirements of formation flying. Therefore, a new method of formation satellites autonomous navigation is proposed. The novel approach utilizes the pulsars timing observations and the measurement of inter-satellite baseline to determine the states of the satellites in a formation. The model of two satellites formation and three satellites formation is also proposed here. The corresponding unscented Kalman filters (UKF) are designed to estimate the position and velocity of the satellites. The method is performed on Matlab in the case of both two satellites formation and three satellites formation. The results show that the new method is feasible and the navigation accuracy is improved obviously. It infers that the proposed method is significant to the navigation of satellites formation.
\end{abstract}

Keywords-formation flying satellites; X-ray pulsars; measurement of inter-satellites baseline UKF filter

\section{INTRODUCTION}

The X-ray pulsar is a kind of high speed spinning neutron star and the pulse information is applied to the whole space. The spacecraft attitude, location, and time navigation information can be provided. With space activities more and more frequent, the number of spacecraft in orbit increasing rapidly. However, most of the spacecraft rely on the ground station, which will increase the burden on the ground station. $\mathrm{X}$-ray Pulsar-based Navigation (XPNAV) is a new astronomical navigation technique that uses the pulse information of X-ray pulsar radiation to obtain the position information of spacecraft.[1] In the spacecraft autonomous navigation XPNAV has a great advantage, first pulsar as a fixed natural objects do not need to maintain, the launch of the signal will not be affected by human damage and interference.[2] Second, the pulse signal can be applied to the entire space, which can provide spacecraft space, attitude and time and other navigation information. Finally, the spacecraft can use pulsars to achieve autonomous navigation, reduce the burden on the ground control system, and reduce the cost of operating spacecraft. But it is a fact that the X-ray pulsar is far away from the solar system, the signal is very weak when it reaches a spacecraft and it is difficult to achieve a better navigation accuracy compared with the GNSS.

To improve the accuracy of autonomous navigation based on x-ray pulsar for space exploration, current research on pulsars navigation mainly focusses on signal source generating, photon detection and counting, the establishment and solution of the navigation model. A prototype for X-ray photon counting detectors for pulsar navigation is developed ${ }^{[3]}$. Light quantum detection based XPNAV hardware in-the-loop simulation system was designed and implemented. Through analyzing the X-ray pulsar signal, the theoretical model of the system was established. The Renyi entropy method of pulsar profile cumulation was proposed, and the X-ray pulsar signal was generated ${ }^{[4]}$.

Navigation method is one of the key technologies to realize satellite formation ${ }^{[5]}$. Satellite status and attitude determination are the prerequisites for satellites to achieve cooperative work. For the formation of satellite navigation, most scholars will focus on GPS technology ${ }^{[6][7]}$ or GPS-like technology, inter-satellite baseline measurement technology and visual-based inter-satellite relative navigation technology. $\mathrm{X}$-ray pulsars can periodically radiate electromagnetic waves when they rotate, which can provide position and time information for the spacecraft and are therefore referred to as future GPS replacements ${ }^{[8][9]}$. The formation of satellite X-ray pulsar navigation is still in the early stages of research, so this paper gradually formed the satellite and X-ray pulsar related field research results, the formation of satellite X-ray pulsar autonomous navigation method in-depth study.

In this paper a new method of formation satellites ${ }^{[10]}$ autonomous navigation is proposed, which make use of pulsars timing observations and the measurement of inter-satellites baseline ${ }^{[11][12]}$ to determine the states of formation satellites. A novel astronomical application for formation flying small satellites is proposed to implement a distributed space mission using formation flying of small satellites. ${ }^{[13]}$

Because of the great advantages of pulsars for spacecraft navigation, some work and research have promoted the improvement of the pulsar-based navigation technology. A new binary timing model added into the navigation. ${ }^{[14]}$ An algorithm using X-ray phase measurements and GNSS pseudo-range for GEO navigation is presented. ${ }^{[15]}$ Utilizing $\mathrm{X}$-ray pulsars for relative navigation suggested by Amir Abbas Emadzadeh ${ }^{[16]}$ and a system measurement model by combining relative pulsar-based and inter-satellite range measurements is built. ${ }^{[17]}$ Although research on pulsars navigation has achieved a lot, but the current accuracy of Pulsar-based navigation can't meet the needs of aerospace. From the perspective of improving Pulsar-based navigation precision this paper proposed a new method making full use of use of space vector 
information of formation satellites to realize the autonomous navigation.

In this paper, a fast phase comparison of pulsar signal is proposed, at the same time a new method to add the angle between the inter-satellite baseline is presented and the improvements are as follows: In the double stars navigation, adding the angle between the baseline and the pulsar direction; while in three stars navigation, introducing the angle between satellite plane involving three stars and the direction of the pulsar. This method which considers the formation satellite as a whole to fully utilize the space vector information by means of a UKF filter, to some extent improve the navigation accuracy.

\section{THE BASIC PRINCIPLE OF XNAV}

$\mathrm{X}$-ray pulsar radiation photon energy concentration is convenient for small device for detection and processing, which is the primary choice for autonomous navigation. X-ray pulsar autonomous navigation (XPNAV) records the X-ray photon time of arrival (TOA) pulsar radiation, while performing a series of signal processing to obtain spacecraft status information.

\section{A Geometric Principle of X - ray Pulsar Navigation}

In order to improve the accuracy of pulsar navigation, it is necessary to use X-ray pulse contour with high signal-to-noise ratio and ensure the stability of the pulse timing model. Therefore, XPNAV basic theory and mathematical model are based on the framework of solar system centroid reference system. Figure I shows the geometrical schematic of X-ray pulse navigation in the solar system centroid reference system.

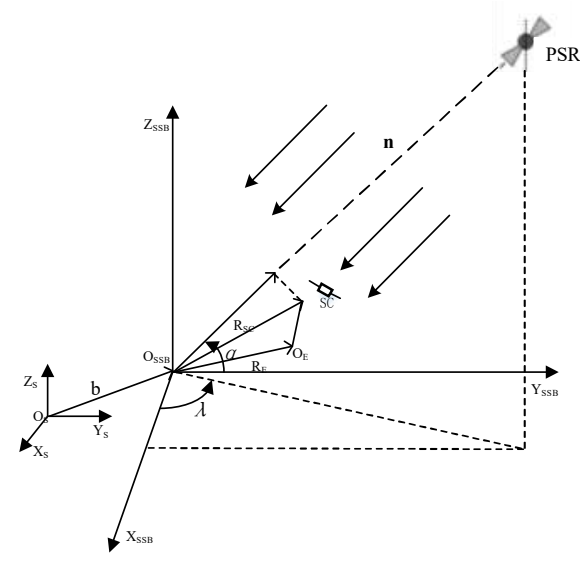

FIGURE I. FTHE GEOMETRIC RELATIONSHIPS BETWEEN PULSAR, EARTH, SUN AND SPACECRAFT IN SSB

As shown in Figure 2.1, select the solar system centroid $\mathrm{O}_{\mathrm{SSB}}$ for the coordinates of origin, the $\mathrm{X}_{\mathrm{SSB}}$ axis direction points to the vernal equinox defined by the standard epoch J2000.0, According to the right hand rule to determine the direction of $\mathrm{Y}_{\mathrm{SSB}}$, a solar system centroid reference system $\mathrm{O}_{\mathrm{SSB}}-\mathrm{X}_{\mathrm{SSB}} \mathrm{Y}_{\mathrm{SSB}} Z_{\mathrm{SSB}}$ can be established. Where $\mathrm{O}_{\mathrm{E}}$ is the Earth's center of mass, $\mathrm{O}_{\mathrm{S}}$ represents the sun's center of mass, PSR is the X-ray pulsar, SC is the spacecraft. $\boldsymbol{R}_{\boldsymbol{S} C}$ and $\boldsymbol{R}_{\boldsymbol{E}}$ are the three-dimensional position coordinates of the spacecraft and the earth in the solar system centroid reference system, $\boldsymbol{R}_{\boldsymbol{S C} / \boldsymbol{E}}$ represents the position vector of the spacecraft relative to the Earth in the solar system centroid reference system. $\boldsymbol{Q}$ is the projection point of the $\boldsymbol{R}_{S C}$ in the direction of the pulsar, $\boldsymbol{n}$ is the direction of the pulsar radiation signal, that is, the pulse vector of the pulsar radiation, and $\alpha$ and $\lambda$ denote the red and declination of the pulsar in the solar system centroid reference system. There are:

$$
\boldsymbol{n}=[\cos \alpha \cos \lambda \cos \alpha \sin \lambda \sin \alpha]
$$

Since the distance between the pulsars and the solar system is very remote, up to tens of thousands of light years, so in the entire solar system $\boldsymbol{n}$ can be seen as a constant $n$. It is observed after a long time in the pulsar information database, easy access at any time. The pulsars radiate the pulsed beams in the universe along the direction of the poles in each cycle. After the X-ray detectors and the atomic clocks are arranged on the spacecraft, the detectors will receive a pulse signal, and can use the satellite atomic clock to record the pulse signal TOA and $t_{s c}$. At the same time, according to the pulsar timing model, it can accurately predict the same pulse signal to reach the solar system centroid time $t_{S S B}$. The basic equations of XPNAV can be obtained by the relationship between $O_{S S B}$, spacecraft and $\boldsymbol{Q}$ given in Figure 2.1:

$$
t_{S S B}-t_{s c}=\frac{1}{c} \boldsymbol{n} \bullet \boldsymbol{R}_{S C}+\delta_{S C}
$$

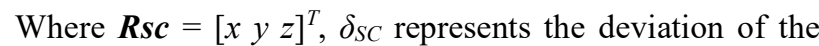
satellite-borne atomic clock; If could more than four X-ray pulsars can be achieved, and then through a number of digital means, the location of the spacecraft and the clock deviation can be estimated. It can be seen, XPNAV is similar to geometry and modern satellite.

In order to improve the navigation accuracy in the practical engineering application, the Kalman filter and the spacecraft orbit dynamic model are combined to estimate the state of the spacecraft. This will not only reduce the estimated error, but also can reduce the detection of more than four pulsars requirements.

\section{B X-ray Pulsar Pulse Time of Arrival Measurement}

In XPNAV, the pulse time of arrival is the basic view required to determine the state of the spacecraft. Only by the different measuring TOA and forecasting TOA, the time delay from the solar system centroid along the pulsar radiation direction to reach the spacecraft can be obtained, then completing the estimation of the spacecraft's state.

The measurement of the arrival time of the pulse includes obtaining both the integer period and the fractional cycle, where the integer period can be obtained by resolving the integer ambiguity method and the fractional part can be obtained by multiplying the phase deviation by the pulsar period. The phase deviation of the pulsars can be calculated by calculating the phase deviation of the measured contour and the standard contour in a pulse period, the so-called phase measurement.

For the same X-ray pulsar, assuming that the standard 
contour $s(t)$ is known, $p(t)$ is the measured profile accumulated over a period of time, there is a relationship between the two:

$$
p(t)=a+b \cdot s(t-D)+g(t)
$$

Where $a$ is the constant deviation of the measurement profile and the standard contour; $b$ is the pulse contour scale transformation factor; $D$ is the time delay amount; $g(t)$ represents the random noise. The final purpose of phase comparison is to find the values of $a, b$, and $D$.

At present, the more classical phase comparison methods include classical time domain correlation method, Taylor-FFT algorithm and TOA maximum likelihood measurement method ${ }^{[18]}$ using bispectrum measurement time delay method $^{[19]}$, nonlinear least squares method[20] and pulse star delay quantity consistency Estimation method ${ }^{[21]}$ and so on.

\section{X-ray pulsed Star Photon Time of Arrival Conversion}

In XPNAV, the use of the spacecraft to carry the atomic clock to measure the X-ray photon to reach the spacecraft time, can be obtained under the coordinates of the spacecraft inherent. The pulsar navigation model is based on the solar system centroid cube reference system based on the establishment, can only use TCB or TDB as a time scale. In order to compare the measured TOA and the predicted TOA at the same time scale, it is necessary to convert the photon to the spacecraft when it is inherently converted to TCB.

Taking the Earth satellite as an example to describe the TOA transformation model. The real space is a curved Riemannian space. The earth centroid reference system and the solar system centroid reference system are established when the motion state of the Earth orbit satellite is described in the large scale space-time range. Although the geosphere reference system and the solar system centroid reference system can express the transformation relationship between the inherent and the coordinates, the XPNAV system uses the conversion when the inherent coordinate system coordinates the solar system. In the whole solar system, although the sun's highest quality, gravity the strongest, but the sun's gravitational radius and its physical radius of the ratio is very small (10-6order of magnitude), it can be the solar system as a gravitational field, the space-time is similar to straight $O$ of the Minkowski space. Therefore, the basic relationship between $\tau$ and TCB can be established in the solar system centroid reference frame.

In the solar system centroid reference frame, set spacecraft space-time coordinates $\left(c_{t}, x_{i}\right)$, then use the complete Newtonian gauge that time and space interval:

$$
d s^{2}=-c^{2} d \tau^{2}=\left(-1+\frac{2 \omega}{c^{2}}-\frac{2 \omega^{2}}{c^{4}}\right) c^{2} d \tau^{2}+\left(-\frac{4 \omega^{i}}{c^{3}}\right) c d t d x^{i}+\delta_{i j}\left(1+\frac{2 \omega}{c^{2}}\right) d x^{i} d x^{j}
$$

In equation (4), $\tau$ is inherent time, $t$ is the coordinate time at the time scale of $\mathrm{TCB} ; \omega 、 \omega_{i}$ represent the scalar gravitational sum and the vector gravitational sum, which is the space-time position of the spacecraft in the solar system's reference frame, and the other planets caused by the earth and the solar system.
Because the spacecraft's three-dimensional rate can be expressed as equation (5):

$$
v=\sqrt{\left(\frac{d x}{d t}\right)^{2}+\left(\frac{d y}{d t}\right)^{2}+\left(\frac{d z}{d t}\right)^{2}}
$$

Bring equation (5) into equation (4) and only retain the $\frac{1}{c^{2}}$ item, can be obtained:

$$
d t=\left(1+\frac{\omega}{c^{2}}+\frac{v^{2}}{2 c^{2}}\right) d \tau
$$

Integrate (6) can be obtained from the inherent time to TCB time scale relationship:

$$
t-t_{0}=\int_{t_{0}}^{t} d t=\int_{t_{0}}^{t}\left(1+\frac{\omega}{c^{2}}+\frac{v^{2}}{2 c^{2}}\right) d \tau=\left(t-t_{0}\right)+\int_{t_{0}}^{t}\left(\frac{\omega}{c^{2}}+\frac{v^{2}}{2 c^{2}}\right) d \tau
$$

The results show that the time scale conversion error of the formula (7) is generally not more than $10^{-12} \mathrm{~s}$, which can meet the X-ray pulse navigation TOA conversion requirements. It can be seen that if the time transition model exists $1 \mu \mathrm{s}$ in the determination of the track will produce $300 \mathrm{~m}$ position errors, therefore, the establishment of high-precision conversion model, relativistic effect must be considered.

Equation (8) can be obtained by equation (6):

$$
c^{2} d t^{2}=\frac{1+\frac{2 \omega}{c^{2}}}{1-\frac{2 \omega}{c^{2}}}\left(d x^{2}+d y^{2}+d z^{2}\right)
$$

Binomial expansion, retain $\frac{1}{c^{2}}$ item:

$$
c d t=\left(1+\frac{2 \omega}{c^{2}}\right) \sqrt{d x^{2}+d y^{2}+d z^{2}}
$$

For single X-ray pulsar pulse signal, the time delay of its photons from the pulsar to the spacecraft is related to the path distance of the photon motion. Figure II shows the basic process of the X-ray pulsed radiation in the BCRS reaching the Earth's orbiting spacecraft. Where PSR is pulsar, SC is a spacecraft , $O_{S S B}$ represents the solar system centroid, $E$ is the earth's center of mass; $Q_{p k}$ is the other planetary centroids of the solar system, $b, p$ and $D$ denote the position vector of the centroid of the sun, the centroid of the earth and the other centroids of the solar system in the BCRS, respectively, $b_{k}, p_{k}$ and $D_{k}$ represent the vectors of the other planets of the solar system in the BCRS coordinate system, respectively, to SSB, spacecraft and pulsars, $n_{S}, n_{S S B}$ and $n_{S C}$ represent the angular position vectors from the solar system centroid, SSB, and the spacecraft to the pulsars. 


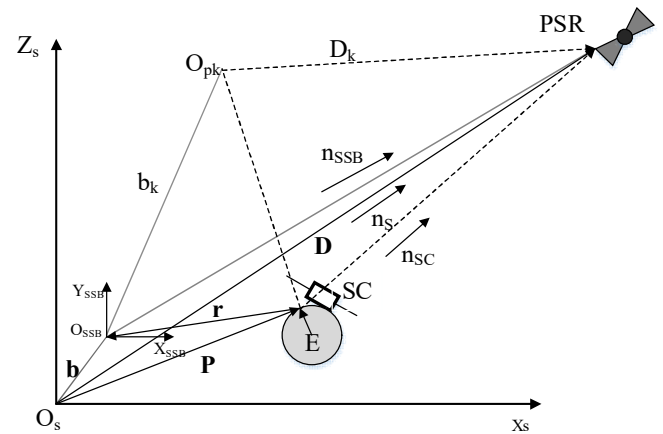

FIGURE II. PATHWAYS OF PULASAR RADIATION X-RAY PHOTONS REACHING THE EARTH'S ORBITAL SPACECRAFTF

At the time scale of the TCB, it is assumed that the pulsar emits the Nth pulse $X_{s}$ signal at $t_{s}$ time, the SSB arrives at $t_{S S B}$, and the time at which the spacecraft's detection device receives is $t_{S C}$. In the $t_{T}$ to $t_{S C}$ time period and $t_{T}$ to $t_{S C}$ time period respectively on the equation (9), and subtraction after the spacecraft relative to the SSB pulse arrival time mathematical conversion model:

$$
\begin{aligned}
& t_{S S B}-t_{S C}=\frac{1}{c} \mathbf{n}_{\mathrm{SSB}} \cdot(\mathbf{D}-\mathbf{b})-\frac{1}{c} \mathbf{n}_{\mathrm{SC}} \cdot(\mathbf{D}-\mathbf{p})- \\
& \sum_{k=1}^{m_{p}} \frac{2 \mu_{k}}{c^{3}} \ln \left|\frac{\mathbf{n}_{\mathrm{SSB}} \cdot \mathbf{b}_{\mathbf{k}}+b_{k}}{\mathbf{n}_{\mathrm{SSB}} \cdot \mathbf{D}_{\mathbf{k}}+D_{k}}\right|+\sum_{k=1}^{m_{p}} \frac{2 \mu_{k}}{c^{3}} \ln \left|\frac{\mathbf{n}_{\mathrm{SSB}} \cdot \mathbf{p}_{\mathbf{k}}+p_{k}}{\mathbf{n}_{\mathrm{SSB}} \cdot \mathbf{D}_{\mathbf{k}}+D_{k}}\right|+ \\
& \frac{2 \mu_{s}^{2}}{c^{5} D_{y}^{2}}\left\{\begin{array}{l}
\mathbf{n}_{\mathrm{SSB}} \cdot(\mathbf{D}-\mathbf{b})\left[\left(\frac{\mathbf{n}_{\mathrm{SSB}} \cdot \mathbf{D}}{D}\right)^{2}+1\right]+2\left(\mathbf{n}_{\mathrm{SSB}} \cdot \mathbf{D}\right)\left(\frac{b}{D}-1\right) \\
+D_{y}\left[\arctan \left(\frac{p_{x}}{D_{x}}\right)-\arctan \left(\frac{D_{x}}{D_{y}}\right)\right]
\end{array}\right\}- \\
& \frac{2 \mu_{s}^{2}}{c^{5} D_{y}^{2}}\left\{\begin{array}{l}
n_{S C} \cdot(\mathbf{D}-\mathbf{p})\left[\left(\frac{\mathbf{n}_{\mathrm{SSB}} \cdot \mathbf{D}}{D}\right)^{2}+1\right]+2\left(\mathbf{n}_{\mathrm{SC}} \cdot \mathbf{D}\right)\left(\frac{p}{D}-1\right) \\
+D_{y}\left[\arctan \left(\frac{p_{x}}{D_{y}}\right)-\arctan \left(\frac{D_{x}}{D_{y}}\right)\right]
\end{array}\right\}
\end{aligned}
$$

Where $P=[p x, p y, p z]^{\mathrm{T}}, D=[D x, D y, D z]^{\mathrm{T}}$. And because the entire solar system pulsar direction vector almost unchanged, that can be seen as a constant. That is,

$$
\begin{aligned}
t_{S S B}-t_{S C} & =\frac{\mathbf{n} \cdot \mathbf{r}}{c}+\frac{1}{2 c D_{0}}\left[(\mathbf{n} \cdot \mathbf{r})^{2}-r^{2}+2(\mathbf{n} \cdot \mathbf{b})(\mathbf{n} \cdot \mathbf{r})-2(\mathbf{b} \cdot \mathbf{r})\right]+ \\
& \frac{2 \mu_{s}}{c^{3}} \ln \left|\frac{\mathbf{n} \cdot \mathbf{r}+\mathbf{n} \cdot \mathbf{b}+\mathbf{r}+\mathbf{b}}{\mathbf{n} \cdot \mathbf{b}+\mathbf{b}}\right|
\end{aligned}
$$

The first term on the right side of the equation (11) represents the time delay caused by the geometric distance of the spacecraft relative to the SSB, called the Dopper delay and the second term indicates the time delay in which the X-ray photon arrives in parallel to the solar system. Generally the first two items are called Roemer delay. The third term indicates the delay caused by the bending of the light under the action of the solar gravitational field, called the Shapiro delay.

\section{SATEllite NAVigation Model}

\section{A Baseline Definition}

The "inter-satellite baseline" indicates that, when satellite control is achieved, the baseline is usually represented by a connection between two satellite centroids in a given coordinate system. ${ }^{[22]}$ The representation of the baseline in different coordinate systems is also different, as shown in Figure III. In the Cartesian coordinate system, the inter-satellite baseline vector can be represented by its projection in the three-dimensional Cartesian coordinate system, that is, $b=(x, y, z)^{\mathrm{T}}$; in the spherical coordinate system, it can be expressed as $b=(B, \theta, \varphi)^{\mathrm{T}}$, where $B$ is the length of the inter-satellite baseline, $\theta$ is the azimuth angle in the spherical coordinate system, and $\varphi$ is the height angle. The conversion of these two methods is shown in Equation (12)

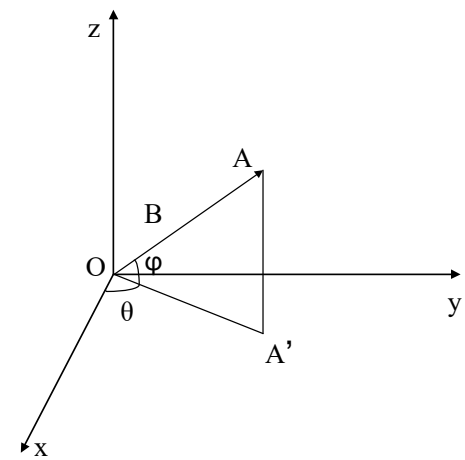

FIGURE III. REPRESENTATION OF INTER-SATELLITE OF BASELINES IN DIFFERENT COORDINATE SYSTEMS

$$
\left\{\begin{array}{l}
B=\sqrt{x^{2}+y^{2}+z^{2}} \\
\varphi=\arcsin \left(\frac{z}{\sqrt{x^{2}+y^{2}+z^{2}}}\right) \\
\theta=\arctan \left(\frac{y}{z}\right)
\end{array}\right.
$$

From (12), the baseline measurement is the means of obtaining the baseline value in the top coordinate system by means of direct measurement or parameter estimation. At present, there are two methods that can be used: first the inter-satellite baseline information is obtained by comparing the state values of the satellites, and the status values of the individual satellites can be obtained by means of measurement or estimation. ${ }^{[23]}$ Second the use of a measurement method to directly measure or estimate the information. ${ }^{[24][25]}$ Since the ultimate goal of the method presented in this paper is to estimate the absolute state of the formation satellites, that is, the state of the formation satellites is unknown. Therefore, the relative state measurement method must be used to obtain the length of the inter-satellite baseline information. Considering the measurement elements, measurement accuracy and data rate of various methods, the laser measurement method is used to obtain the inter-satellite baseline length of formation satellites.

\section{B Double Satellites Formation Navigation Model}

The traditional X-ray pulsar-based navigation method (also known as pulse chronograph) uses only three pulsar photon information to achieve autonomous navigation of the spacecraft. On the basis of the traditional method, this paper presents a new binary star formation autonomous navigation method, which is based on the observation of pulsar time, and 
increases the angle between the baseline of the formation satellites and the radiation direction of the pilot pulsars Observations to obtain more accurate satellite navigation information. In the case of two satellites, as the satellites continue to move, the direction of the formation of the satellite baseline is constantly changing, so that the angle between the baseline of the formation satellites and the direction of the radiation of the pilot pulsars is changing at all times. The angle as a measure is technically feasible. The geometric model of the method is shown in Figure IV.

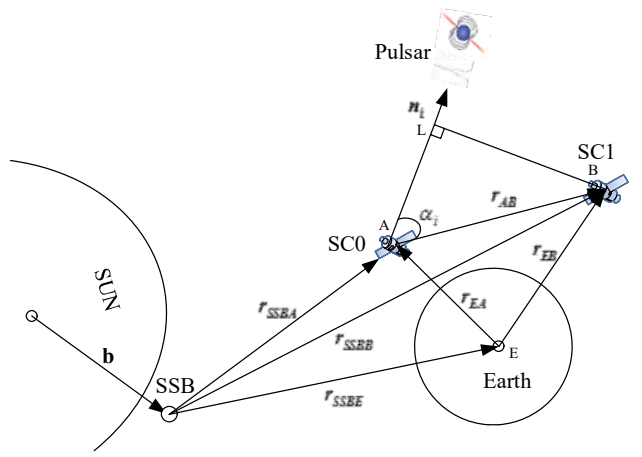

FIGURE IV. DOUBLE STAR FORMATION GEOMETRIC MODEL

In Figure IV, $b$ is the position coordinate of the sun in the solar system centroid reference frame, $r_{E S S B}$ is the position coordinates of the earth in the solar system centroid reference frame, $r_{s s b 0}$ is the position vector of the formation satellite SC0 (reference satellite) in the solar system centroid reference frame, $r_{s s b 1}$ is the position vector of the formation satellite SC1 (accompanied satellite) in the solar system centroid reference frame, and $r$ is the baseline vector between the satellite SC0 and the satellite $\mathrm{SC} 1$, which direction is directed by SC0 to SC1, and then:

$$
\mathbf{r}=\mathbf{r}_{\text {ssb1 }}-\mathbf{r}_{\text {ssb0 }}
$$

Use $\alpha_{i}$ to represent the angle between the baseline of the formation satellites and the pulsar radiation direction vector, and $n_{i}$ for the pulsar radiation direction vector. From Figure 3.2, $\alpha_{i}$ can be expressed as:

$$
\alpha_{i}=h(\mathbf{r}, t)=\arccos \frac{\mathbf{n}_{\mathbf{i}} \cdot \mathbf{r}}{|\mathbf{r}|}+\mathbf{n}_{\mathbf{a i}}
$$

Where $n_{\alpha i}$ denotes the observed noise; $n_{i} . r$ denotes the projection of the baseline between the reference satellite and the accompanying satellite in the ith pulsar radiation; $|r|$ represents the baseline length of the formation satellite; the range of $\alpha_{i}$ varies from 0 to $2 \pi$. Put equation (13) into the equation (14) available:

$$
\alpha_{i}=\arccos \frac{\mathbf{n}_{\mathbf{i}} \cdot\left(\mathbf{r}_{\mathrm{ssb} 1}-\mathbf{r}_{\mathrm{ssb0}}\right)}{|\mathbf{r}|}+\mathbf{n}_{\mathrm{ai}}=\arccos \frac{\mathbf{n}_{\mathbf{i}} \cdot \mathbf{r}_{\mathrm{ssb1}}-\mathbf{n}_{\mathbf{i}} \cdot \mathbf{r}_{\mathrm{ssb0}}}{|\mathbf{r}|}+\mathbf{n}_{\mathrm{ai}}
$$

According to the pulsar basic principle described in Section 2 , if $t_{\mathrm{DOA}}$ is used to represent the propagation delay between the SCO and SC1 of the X-ray pulsar signal. There are

$$
\mathbf{n}_{\mathbf{i}} \cdot \mathbf{r}_{\text {ssb1 }}-\mathbf{n}_{\mathbf{i}} \cdot \mathbf{r}_{\text {ssb } 0}=c \cdot t_{D O A}
$$

Where $c$ is the speed of light, and equation (16) is substituted into equation (15):

$$
\alpha_{i}=\arccos \frac{c \cdot t_{D O A}}{|\boldsymbol{r}|}+\boldsymbol{n}_{a i}
$$

In the above equation, the value of $t_{\mathrm{DOA}}$ is obtained by taking the X-ray pulse signal to $\mathrm{SC} 0$ and the time difference of $\mathrm{SC} 1$. The baseline length $|r|$ can be obtained by laser measurement, and the value of $\alpha_{i}$ can be calculated.

In the implementation of XPNAV, we need three photon information of the pulsars to obtain the absolute position estimation of the satellite. Therefore, this paper also calculates the radiation vector of the three pulsars and the angle of the baseline to obtain more accurate position estimation.

\section{Three Satellites Formation Navigation Model}

In this paper, a new method of pulsar navigation in three satellites formation is presented. On the basis of traditional pulsar navigation, the angle between the plane defined by the three formation satellites and the direction vector of the navigation pulsar is introduced. As a supplemental observation to obtain more accurate satellite navigation information. Figure $\mathrm{V}$ is the geometry of the three satellites formation navigation:

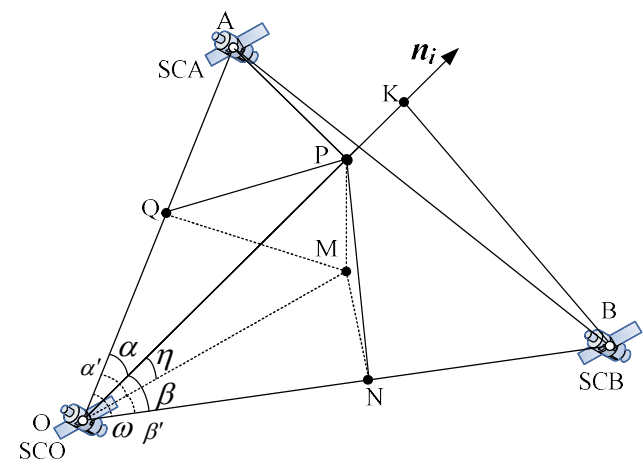

FIGURE V. THREE SATELLITES FORMATION GEOMETRIC MODEL

Where satellite $\mathrm{O}$, satellite $\mathrm{A}$ and satellite $\mathrm{B}$ denote three formation satellites, select satellite $\mathrm{O}$ as reference satellite, satellite A and satellite B as companion satellites. $n_{i}$ represents the pulsar radiation direction vector. Over point $\mathrm{B}$ do $n_{i}$ vertical line at the point $\mathrm{K}$. Over point $\mathrm{A}$ to do $n_{i}$ vertical line at the point $\mathrm{P}$. Over point $\mathrm{P}$ to do the vertical $\mathrm{PQ}$ of OA. Over point $\mathrm{P}$ to do the vertical $\mathrm{PN}$ of $\mathrm{OB}$. Over point $\mathrm{P}$ to do the plane $\mathrm{OAB}$ vertical line, pay the plane at the point $\mathrm{M}$. The angle between the pulse vector of the pulsar radiation and the plane is expressed by $\eta$. The length of the OP is $l$, the length of the QM is $a$, the length of the $\mathrm{MN}$ is $b$, the length of the $\mathrm{ON}$ is $c$, the length of the OQ is $d$, the length of the OM is $e$, and the length of the PM length $h$. Set $\angle P O A$ is $\alpha(\alpha=(0,2 \pi)), \angle P O B$ is $\beta(\beta=(0,2 \pi)), \angle A O B$ is $\omega, \angle P O A$ is projected on the plane $\mathrm{OAB}$ as $\alpha^{\prime}$, and the projection of the plane $\mathrm{OAB}$ is $\beta^{\prime}$, then $\alpha^{\prime}+\beta^{\prime}=\omega$.

In this paper, the three satellites formation pulsar autonomous navigation method is used to realize the high 
precision navigation of formation satellites by using the angle information between the X-ray pulsars' optical travel information and the pulsar radiation direction and the plane defined by the three formation satellites. Assuming that the length of the inter-satellite baseline $\mathrm{OA}, \mathrm{OB}$, and $\mathrm{AB}$ is known (obtained by laser ranging). From the cosine theorem

$$
\cos \omega=\frac{O A^{2}+O B^{2}-A B^{2}}{2 \times O A \times O B}, \omega \leq \pi
$$

At the same time $\alpha=\operatorname{arcos}(\mathrm{OP} / \mathrm{OA})$, where OA is the length of the baseline between the formation satellite $\mathrm{O}$ and the formation satellite $\mathrm{A}$, which can be obtained by laser measurement. The OP length can be calculated by the X-ray pulse signal propagation delay. The delay generated by the propagation of the ray signal between the $\mathrm{O}$ point and the $\mathrm{A}$, then:

$$
l=t_{\mathrm{DOA}} \cdot c
$$

Where $c$ is the speed of light. Calculate $\beta$ with the same.

And it is possible to know $\alpha^{\prime}$ and $\beta^{\prime}$, and $d$ and $c$ can be obtained by the equation (18-19):

$$
\begin{gathered}
\cos \alpha=\frac{l}{O A}=\frac{d}{l} \rightarrow d=\frac{l^{2}}{O A} \\
\cos \alpha=\frac{O K}{O B}=\frac{c}{l} \rightarrow c=\frac{l \times O K}{O B} \\
\alpha^{\prime}+\beta^{\prime}=\omega \\
e=l \cdot \cos \eta
\end{gathered}
$$

According equation (21-23):

$$
\arccos \frac{\frac{l^{2}}{\mathrm{OA}}}{l \times \cos \eta}+\arccos \frac{\frac{l \times \mathrm{OK}}{\mathrm{OB}}}{l \times \cos \eta}=\omega
$$

In equation (24) there is only one unknown quantity, the solution equation is:

$$
\eta=\arccos \sqrt{\left(\left(\frac{l}{\mathrm{OA}}\right)^{2}+\left(\frac{\mathrm{OK}}{\mathrm{OB}}\right)^{2}-2 \times \cos \omega \times \frac{l}{\mathrm{OA}} \times \frac{\mathrm{OK}}{\mathrm{OB}}\right) /\left(1-\cos ^{2} \omega\right)}
$$

Figure VI The Samsung Formation geometric model gives the case where the point $\mathrm{M}$ of the P-point in the plane OAB falls just in the triangle $\mathrm{OAB}$, and there is a case where the projection of the $\mathrm{P}$ point on the plane $\mathrm{OAB}$ falls outside the triangle $\mathrm{OAB}$, Figure 3.5 shows that $\mathrm{M}$ points are outside the triangle $\mathrm{OAB}$, and in this case there are:

$$
\left|\beta^{\prime}-\alpha^{\prime}\right|=\omega
$$

In both cases, the expression of $\eta$ is the same.

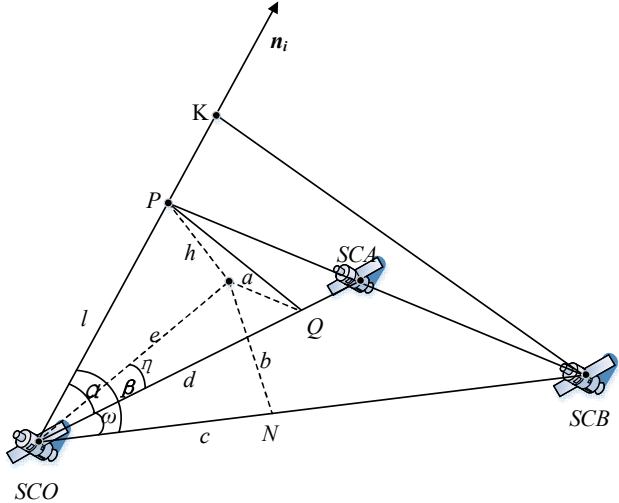

FIGURE VI. ANOTHER EXAMPLE OF THE THREE STAELLITES FORMATION GEOMETRIC MODEL

$$
\eta=\arccos \sqrt{\left(\left(\frac{l}{\mathrm{OA}}\right)^{2}+\left(\frac{\mathrm{OK}}{\mathrm{OB}}\right)^{2}-2 \times \cos \omega \times \frac{l}{\mathrm{OA}} \times \frac{\mathrm{OK}}{\mathrm{OB}}\right) /\left(1-\cos ^{2} \omega\right)}
$$

In the expressions of Eq. (26) and (27) for $\eta$, the length of $\mathrm{OA}, \mathrm{OB}$, and $\mathrm{AB}$ in the expression of $\eta$ can be obtained by means of laser ranging, where $l$ and $\mathrm{OK}$ are the projections of $\mathrm{OA}$ and $\mathrm{OB}$ in the direction of the line of sight of the pulsars, which can be calculated by TDOA. The analysis is easy to change with the change of the satellite position of the formation, which can be used as the supplemental observation of the X-ray pulse navigation to improve the navigation accuracy of the formation satellite.

\section{KALMAN Filter MODEL}

\section{A Unscented Kalman Filtering Model}

Satellite navigation is a dynamic estimation of the satellite state, that is, under certain criteria, the use of the observed signal, the satellite location, speed and acceleration and other information to infer. The purpose of the state estimation is to smooth the past state of the target, filter the current state of the target, and predict the future state of the target. In the satellite navigation problem, the system state equation and the measurement equation are not linear, so this paper chooses to use the UKF filter to achieve the prediction of the formation satellite status, compared to EKF, UKF can better solve the nonlinear system state estimation problem.

\section{B Dynamic Model of Satellite Orbit}

At some point the satellite state vector $X$ can use six parameters to complete the accurate description of the spacecraft's orbital state. The spacecraft's orbital state contains three position vectors $r=r_{S C}=\{r x, r y, r z\}^{\mathrm{T}}$ and three velocity vectors $v=v_{\mathrm{SC}}=\{v x, v y, v z\}^{\mathrm{T}}$. The satellite state vector can be expressed as:

$$
\mathbf{X}=\left[\begin{array}{l}
\mathbf{r} \\
\mathbf{v}
\end{array}\right]
$$

The dynamic model of a nonlinear system can be expressed as a state vector: 


$$
\dot{\mathbf{X}}=f(\mathbf{X}(t), t)+\mathbf{w}(t)
$$

Where $\mathrm{f}$ represents the nonlinear dynamic function of the state vector and $w(t)$ represents the state noise. Since the acceleration $a$ is the derivative of the velocity versus time, the velocity is the derivative of the position versus time. After ignoring noise, the derivative of the state vector can be expressed as:

$$
\dot{\mathbf{X}}=f(\mathbf{X}(t), t)=\left[\begin{array}{c}
\dot{\mathbf{r}} \\
\dot{\mathbf{v}}
\end{array}\right]=\left[\begin{array}{l}
\mathbf{v} \\
\mathbf{a}
\end{array}\right]
$$

On both sides carry on the integral operation of the equation (31):

$$
\mathbf{X}(t)=\mathbf{X}\left(t_{0}\right)+\int_{t_{0}}^{t} f(\mathbf{X}(\tau), \tau) d \tau
$$

Once the initial state of the satellite is known:

$$
\mathbf{X}\left(t_{0}\right)=\mathbf{X}_{0}=\left[\begin{array}{l}
\mathbf{r}_{0} \\
\mathbf{v}_{\mathbf{0}}
\end{array}\right]
$$

And its acceleration can be calculated, then the state of motion of the satellite can be solved. If the expression of equation (32) can be obtained, the state of the satellite at any subsequent time can be automatically calculated. However, due to the impact of a number of high-order, it is difficult to obtain accurate resolution of the satellite state. Therefore, the initial state of the satellite and the numerical integration of the kinetic equation are used to obtain the state information of the satellite at a certain time in the future.

\section{Double Satellites Unscented Kalman Filter Model}

The method presented in this paper combines the X-ray time-observation information and the information of the angle between the formation satellite base and the X-ray pulsar radiation direction to obtain a more accurate state estimation than the X-ray pulse timing observation. The method uses the UKF filter to obtain an estimate of the formation satellite status, the system's state quantity is:

$$
X=\left[\begin{array}{llll}
r_{0} & v_{0} & r_{1} & v_{1}
\end{array}\right]^{T}
$$

Where $\boldsymbol{r}_{\boldsymbol{0}}, \boldsymbol{v}_{\boldsymbol{0}}$ and $\boldsymbol{r}_{\boldsymbol{1}}, \boldsymbol{v}_{\boldsymbol{1}}$ represent the position and velocity of the reference satellite and concomitant satellite in the solar system centroid celestial coordinate system.

Assuming that the observed amount $\boldsymbol{Z}_{\boldsymbol{I}}$ represents the angle between the X-ray pulse radiation direction and the formation satellite base, with $\alpha_{i}$, the observed noise is $V_{I}=\left[n_{\alpha i}\right]$, then the measurement equation can be expressed as:

$$
Z_{I}=h_{1}[x(t), t]+V_{I}(t)
$$

where:

$$
h_{1}[\mathbf{x}(t), t]=\arccos \frac{\mathbf{n}_{\mathbf{i}} \cdot \mathbf{r}}{|\mathbf{r}|}=\arccos \frac{c \cdot T D O A}{|\mathbf{r}|}
$$

Where $\boldsymbol{r}=\boldsymbol{r}_{s s b 1}-\boldsymbol{r}_{s s b 1}$ is the baseline vector of the formation satellites, $\boldsymbol{n}_{\boldsymbol{i}}$ is the pulsar radiation direction vector, $\boldsymbol{n}_{\boldsymbol{i}} \cdot \boldsymbol{r}$ can be obtained by pulse arriving at the reference satellite and the satellite time difference (TDOA), $|\boldsymbol{r}|$ can be obtained by baseline measurements. And $n_{\alpha i}$ represents the combined error of the angle measurement caused by the formation of the satellite baseline and the detection of the arrival time of the pulse, which is the white noise with a mean of 0 and a variance of $R_{1}$.

In X-ray pulsar-based navigation, it is necessary to convert the time detected at the satellite to the time at which the pulse reaches the $\mathrm{SSB}$. The transfer equation is:

$$
\begin{aligned}
t_{S S B}^{i}-t_{S C}^{i}= & H\left(\boldsymbol{r}_{S S B}, \boldsymbol{n}_{i}, D_{0}, \boldsymbol{b}\right)=\frac{\boldsymbol{n}_{i} \cdot \boldsymbol{r}_{S S B}}{c}+\frac{1}{2 c D_{0}}\left[\left(\boldsymbol{n}_{\boldsymbol{i}} \cdot \boldsymbol{r}_{S S B}\right)^{2}-r_{S S B}{ }^{2}+\right. \\
& \left.2\left(\boldsymbol{n}_{i} \cdot \boldsymbol{b}\right)\left(\boldsymbol{n}_{\boldsymbol{i}} \cdot \boldsymbol{r}_{S S B}\right)-2\left(\boldsymbol{b} \cdot \boldsymbol{r}_{S S B}\right)+2\left(\boldsymbol{n}_{\boldsymbol{i}} \cdot \boldsymbol{V} \Delta t_{N}\right)\left(\boldsymbol{n}_{\boldsymbol{i}} \cdot \boldsymbol{r}_{S S B}\right)\right]+ \\
& \frac{2 \mu_{S U n}}{c^{3}} \ln \left|\frac{\boldsymbol{n}_{\boldsymbol{i}} \cdot \boldsymbol{r}_{S S B}+r_{S S B}}{\boldsymbol{n}_{\boldsymbol{i}} \cdot \boldsymbol{b}+b}+1\right|
\end{aligned}
$$

Where $i$ denotes the ith pulse star, $\boldsymbol{r}_{S S B}$ denotes the position of the satellite in the BCRS, $\boldsymbol{V}$ denotes the moving speed of the pulsar, and $\Delta t_{N}=t_{N}-t_{0}$ denotes the time interval from the initial time to the transmission of the Nth pulse. Since $\boldsymbol{V}$ is small, and $D_{0}>>\boldsymbol{V} \Delta t, \boldsymbol{V} \Delta t$ is negligible.

Let $\boldsymbol{Z}_{2}=c \cdot\left(t_{S S B}-t_{S C}^{i}\right)=\Delta \boldsymbol{T}^{\boldsymbol{i}}$ represent the observed observation of the pulsar timing, and the observed noise is $\boldsymbol{V}_{\mathbf{2}}$ $=\left[n_{t i}\right]$

$$
Z_{2}=h_{2}[x(t), t]+V_{2}(t)
$$

where,

$$
\begin{aligned}
h_{2}[\boldsymbol{X}(t), t] & =\frac{1}{2 D_{0}}\left[\left(\boldsymbol{n}_{\boldsymbol{i}} \cdot \boldsymbol{r}_{S S B}\right)^{2}-r_{S S B}{ }^{2}+2\left(\boldsymbol{n}_{i} \cdot \boldsymbol{b}\right)\left(\boldsymbol{n}_{\boldsymbol{i}} \cdot \boldsymbol{r}_{S S B}\right)-2\left(\boldsymbol{b} \cdot \boldsymbol{r}_{S S B}\right)\right]+ \\
& \boldsymbol{n}_{\boldsymbol{i}} \cdot \boldsymbol{r}_{S S B}+\frac{2 \mu_{S u n}}{c^{2}} \ln \left|\frac{\boldsymbol{n}_{\boldsymbol{i}} \cdot \boldsymbol{r}_{S S B}+r_{S S B}}{\boldsymbol{n}_{\boldsymbol{i}} \cdot \boldsymbol{b}+b}+1\right|
\end{aligned}
$$

$V_{2}$ is the observed noise with mean 0 and variance $\sigma_{i}^{2}$. The standard deviation $\sigma_{i}$ can be obtained by Taylor's formula.

In the realization of double satellites formation:

$$
\dot{X}=f(X(t), t)+\omega(\mathrm{t})=\left[\begin{array}{c}
\dot{\boldsymbol{r}}_{0} \\
\dot{\boldsymbol{v}}_{0} \\
\dot{\boldsymbol{r}}_{1} \\
\dot{\boldsymbol{v}}_{1}
\end{array}\right]=\left[\begin{array}{c}
\boldsymbol{v}_{0} \\
\boldsymbol{a}_{0} \\
\boldsymbol{v}_{1} \\
\boldsymbol{a}_{1}
\end{array}\right]
$$

It is possible to predict the state of the satellites at any time by predicting the initial state.

In summary, the observation of the new method of X-ray pulsar navigation in this paper is as follows: 


$$
\boldsymbol{Z}=\left[\boldsymbol{Z}_{1}, \boldsymbol{Z}_{2}\right]=\left[\alpha_{1}, \alpha_{2}, \alpha_{3}, \Delta \boldsymbol{T}_{0}^{1}, \Delta \boldsymbol{T}_{0}^{2}, \Delta \boldsymbol{T}_{0}^{3}, \Delta \boldsymbol{T}_{1}^{1} \Delta \boldsymbol{T}_{1}^{2} \Delta \boldsymbol{T}_{1}^{3}\right]^{\mathrm{T}}
$$

Where:

$$
\boldsymbol{Z}=\boldsymbol{H} \boldsymbol{X}+\boldsymbol{N}(\boldsymbol{t})
$$

$\boldsymbol{H}$ is the measurement matrix, $\boldsymbol{N}(\boldsymbol{t})$ is the observation noise matrix, which can be expressed as:

$$
N(t)=\left[\boldsymbol{n}_{\alpha 1}, \boldsymbol{n}_{\alpha 2}, \boldsymbol{n}_{\alpha 3}, \boldsymbol{n}_{t 1}, \boldsymbol{n}_{t 2}, \boldsymbol{n}_{t 3}, \boldsymbol{n}_{t 1}, \boldsymbol{n}_{t 2}, \boldsymbol{n}_{t 3}\right]
$$

The variance matrix of $N(t)$ can be expressed as

$$
R(t)=\operatorname{diag}\left(\sigma_{a 1}^{2}, \sigma_{a 2}^{2}, \sigma_{a 3}^{2}, \sigma_{1}^{2}, \sigma_{2}^{2}, \sigma_{3}^{2}, \sigma_{1}^{2}, \sigma_{2}^{2}, \sigma_{3}^{2}\right)
$$

Where $\sigma_{\alpha i}^{2}$ is the variance of the observed $\alpha_{i}$, the variance of the chronological observation of the ith pulsar at $\sigma^{2}$.

The state transition equation is:

$$
\dot{X}(k+1)=f(X(t), t)+w(t)=\left[\begin{array}{c}
\dot{\boldsymbol{r}}_{0} \\
\dot{\boldsymbol{v}}_{0} \\
\dot{\boldsymbol{r}}_{1} \\
\dot{\boldsymbol{v}}_{1}
\end{array}\right]=\left[\begin{array}{c}
\boldsymbol{v}_{0} \\
\boldsymbol{a}_{0} \\
\boldsymbol{v}_{1} \\
\boldsymbol{a}_{1}
\end{array}\right]
$$

$\omega(t)$ represents the state noise.

\section{Three Satellites Unscented Kalman Filtering Model}

Three satellites formation navigation method is also in the X-ray pulse timing observation method to introduce new observations, thereby enhancing the navigation accuracy. The angle between the X-ray pulsar radiation direction and the three formation satellites is plotted as the newly introduced observation.

When implementing the UKF filter based on pulsar and baseline information, the state variables is:

$$
X=\left[\begin{array}{llllll}
r_{0} & v_{0} & r_{1} & v_{1} & r_{2} & v_{2}
\end{array}\right]^{T}
$$

Where $\boldsymbol{r}_{\boldsymbol{0}}, \boldsymbol{v}_{0}, \boldsymbol{r}_{1}, \boldsymbol{v}_{\boldsymbol{1}}$ and $\boldsymbol{r}_{2}, \boldsymbol{v}_{\mathbf{2}}$ represent the position and velocity of the reference satellite and concomitant satellite in the solar system centroid celestial coordinate system.

The observation $\boldsymbol{Z}_{I}=\left[\eta_{i}\right]$ indicates the angle between the direction of the pulsar radiation and the plane defined by the formation satellite. The corresponding observed noise is $\boldsymbol{V}_{\boldsymbol{I}}=\left[n_{\eta i}\right]$, and the measurement equation can be expressed as:

$$
Z_{I}=h_{1}[x(t), t]+V_{I}(t)
$$

where:

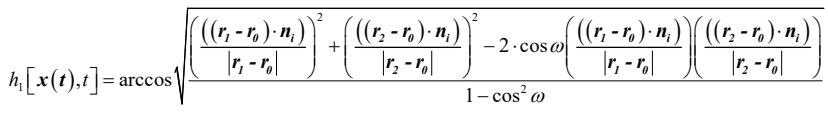

$\boldsymbol{n}_{\boldsymbol{i}}$ is the pulsar radiation direction vector, the values of $\left(\boldsymbol{r}_{1}-\right.$ $\left.\boldsymbol{r}_{\boldsymbol{0}}\right) \cdot \boldsymbol{n}_{\boldsymbol{i}}$ and $\left(\boldsymbol{r}_{2}-\boldsymbol{r}_{\boldsymbol{0}}\right) \cdot \boldsymbol{n}_{\boldsymbol{i}}$ are calculated by the time difference between the arrival of the reference satellite and the satellite. $\boldsymbol{r}_{\boldsymbol{1}}-\boldsymbol{r}_{\boldsymbol{0}} \mid$ and $\left|\boldsymbol{r}_{\boldsymbol{2}}-\boldsymbol{r}_{\boldsymbol{0}}\right|$ are obtained directly from baseline measurements. The definition of $\cos \omega$ is given in Section 4.2. $n_{\eta i}$ is the combined error caused by the formation of the satellite baseline and the detection of the arrival time of the pulse, which is a white noise with a mean of 0 and a variance of $\sigma_{\eta i}^{2}$.

Let $\boldsymbol{Z}_{2}=c \cdot\left(t_{S S B}^{i}-t_{S C}^{i}\right)=\Delta \boldsymbol{T}$ is the observed observation of the pulsar timing, and the observed noise is $\boldsymbol{V}_{2}=\left[n_{t i}\right]$ :

$$
Z_{2}=h_{2}[x(t), t]+V_{2}(t)
$$

Where:

$$
\begin{aligned}
h_{2}[\boldsymbol{X}(t), t]= & \frac{1}{2 D_{0}}\left[\left(\boldsymbol{n}_{\boldsymbol{i}} \cdot \boldsymbol{r}_{S S B}\right)^{2}-r_{S S B}{ }^{2}+2\left(\boldsymbol{n}_{i} \cdot \boldsymbol{b}\right)\left(\boldsymbol{n}_{\boldsymbol{i}} \cdot \boldsymbol{r}_{S S B}\right)-2\left(\boldsymbol{b} \cdot \boldsymbol{r}_{S S B}\right)\right]+ \\
& \boldsymbol{n}_{\boldsymbol{i}} \cdot \boldsymbol{r}_{S S B}+\frac{2 \mu_{S u n}}{c^{2}} \ln \left|\frac{\boldsymbol{n}_{\boldsymbol{i}} \cdot \boldsymbol{r}_{S S B}+r_{S S B}}{\boldsymbol{n}_{\boldsymbol{i}} \cdot \boldsymbol{b}+b}+1\right|
\end{aligned}
$$

$V_{2}$ is the observed noise with mean 0 and variance $\sigma_{i}^{2}$. The standard deviation $\sigma_{i}$ can be obtained by Taylor's formula. In this paper, the observation of the new method of three satellites formation $\mathrm{x}$ - ray pulsar navigation is

$$
\boldsymbol{Z}=\left[\boldsymbol{Z}_{1}, \boldsymbol{Z}_{2}\right]=\left[\eta_{1}, \eta_{2}, \eta_{3}, \Delta \boldsymbol{T}_{0}^{1}, \Delta \boldsymbol{T}_{0}^{2}, \Delta \boldsymbol{T}_{0}^{3}, \Delta \boldsymbol{T}_{1}^{1}, \Delta \boldsymbol{T}_{1}^{2}, \Delta \boldsymbol{T}_{1}^{3}, \Delta \boldsymbol{T}_{2}^{1}, \Delta \boldsymbol{T}_{2}^{2}, \Delta \boldsymbol{T}_{2}^{3}\right]^{T}
$$

Where:

$$
Z=H X+N(t)
$$

$\boldsymbol{H}$ is the measurement matrix, $\boldsymbol{N}(\boldsymbol{t})$ is the observation noise matrix, which can be expressed as:

$$
\boldsymbol{N}(\boldsymbol{t})=\left[n_{\eta 1}, n_{\eta 2}, n_{\eta 3}, n_{t 1}, n_{t 2}, n_{t 3}, n_{t 1}, n_{t 2}, n_{t 3} n_{t 1}, n_{t 2}, n_{t 3}\right]
$$

The variance matrix of $N(t)$ can be expressed as:

$$
\boldsymbol{R}(\boldsymbol{t})=\operatorname{diag}\left(\sigma_{\eta 1}^{2}, \sigma_{\eta^{2}}^{2}, \sigma_{\eta 3}^{2}, \sigma_{1}^{2}, \sigma_{2}^{2}, \sigma_{3}^{2}, \sigma_{1}^{2}, \sigma_{2}^{2}, \sigma_{3}^{2}\right)
$$

Where $\sigma_{\eta i}^{2}$ is the variance of the observed $\eta_{i}$ and the chronological observation of the ith pulse star at $\sigma_{i}^{2}$.

The state transition equation is:

$$
\dot{X}(\boldsymbol{k}+1)=f(X(t), t)+\boldsymbol{w}(\boldsymbol{t})=\left[\begin{array}{c}
\dot{\boldsymbol{r}}_{0} \\
\dot{\boldsymbol{v}}_{0} \\
\dot{\boldsymbol{r}}_{1} \\
\dot{\boldsymbol{v}}_{1} \\
\dot{\boldsymbol{r}}_{2} \\
\dot{\boldsymbol{v}}_{2}
\end{array}\right]=\left[\begin{array}{c}
\boldsymbol{v}_{0} \\
\boldsymbol{a}_{0} \\
\boldsymbol{v}_{1} \\
\boldsymbol{a}_{1} \\
\boldsymbol{v}_{2} \\
\boldsymbol{a}_{2}
\end{array}\right]
$$

$\omega(t)$ is the state noise.

\section{SimUlation AND RESUltS}

\section{A Simulation Parameter Settings}

In the simulation of satellite formation based on X-ray pulsar navigation, it can be divided into two cases: one is the 
reference and companion satellites are moving in the same orbit and the other is in different orbits. In this part, 'traditional' represents data is obtained by the traditional method, 'angle' indicates the method used to add the baseline information to the resulting data.

The parameters of pulsar and orbits utilized for numerical simulation in this paper is shown in Table I and Table II.

TABLE I PARAMETERS OF ORBITS

\begin{tabular}{ccccc}
\hline Orbit name & Semimajor axis $(\mathrm{km})$ & $\begin{array}{c}\text { RAAN } \\
\left({ }^{\circ}\right)\end{array}$ & $\begin{array}{c}\text { Eccentricity } \\
\left({ }^{\circ}\right)\end{array}$ & $\begin{array}{c}\text { Perigee } \\
\left({ }^{\circ}\right)\end{array}$ \\
\hline GPS BIIA-10 & 4164.3 & 224.67 & 0.0116 & 338.24 \\
\hline MEGSAT-1 & 2254.5 & 341.25 & 0.0049 & 281.70 \\
\hline GPS BIIA-4 & 2254.5 & 195.70 & 0.0059 & 75.52 \\
\hline GPS BIIR-3 & 26560.5 & 120.67 & 0.0153 & 64.56 \\
\hline
\end{tabular}

The three X-ray pulsars selected as the observation targets were B0531 + 21, B1937+ 21 and B1821-24, and their related parameters are shown in Table II.

TABLE II PARAMETERS OF PULSAR

\begin{tabular}{cccc}
\hline pulsar name & Right ascension (J2000.0) $(\mathrm{rad})$ & Declination $(\mathrm{J} 2000.0)(\mathrm{rad})$ & Periods $(\mathrm{s})$ \\
\hline $\mathrm{B} 0531+21$ & 1.4597 & 0.3493 & $3.34 \mathrm{e}-2$ \\
\hline $\mathrm{B} 1937+21$ & 5.1472 & 0.3767 & $1.56 \mathrm{e}-3$ \\
\hline B1821-24 & 4.8194 & -0.4341 & $4.41 \mathrm{e}-2$ \\
\hline B Simulation Results of Double Satellites Navigation & Where $p_{1}=20 \mathrm{~m}, \mathrm{p}_{2}=3 \mathrm{~m} / \mathrm{s}$.
\end{tabular}

B Simulation Results of Double Satellites Navigation

In the two satellite formation X-ray pulsar navigation simulation, it is divided into two cases: One is reference satellite and satellite with the same orbit, another is reference satellite and satellite in different orbit. For the first case, the orbit of the formation satellite is referenced to the ground satellite (MEGSAT-1) orbit, and for the second case, the reference satellite is set to the MEGSAT-1 orbit and the satellite is set to GPS orbit (GPS BIIA-10).

It is known that the formation of satellites at the initial moment can be based on its orbital dynamics model using the fourth-order Runge-Kutta method to calculate the position and velocity of the formation satellite at the next moment. The initial error of the formation satellites is:

$$
\delta \mathbf{X}(0)=\left[\begin{array}{l}
\delta \mathbf{X}_{0}(0) \\
\delta \mathbf{X}_{1}(0)
\end{array}\right]
$$

Where $\delta \mathbf{X}_{0}(0)=[0.8 \mathrm{~km} 0.8 \mathrm{~km} \quad 0.8 \mathrm{~km} 5 \mathrm{~m} / \mathrm{s} 5 \mathrm{~m} / \mathrm{s} 5 \mathrm{~m} / \mathrm{s}]^{\mathrm{T}}$ is the position error and velocity at the initial time of the reference satellite, $\delta \mathbf{X}_{1}(0)=[0.8 \mathrm{~km} \quad 0.8 \mathrm{~km} \quad 0.8 \mathrm{~km} 5 \mathrm{~m} / \mathrm{s} 5 \mathrm{~m} / \mathrm{s}$ $5 \mathrm{~m} / \mathrm{s}]^{\mathrm{T}}$ represents the position error and velocity error accompanying the initial time of the satellite. And the position error and velocity error are defined as:

$$
\begin{aligned}
& \delta \mathbf{X}=\sqrt{|\hat{\mathbf{r}}-\mathbf{r}|^{2}} \\
& \delta \dot{\mathbf{X}}=\sqrt{|\hat{\mathbf{r}}-\dot{\mathbf{r}}|^{2}}
\end{aligned}
$$

In the above equation, $\boldsymbol{r}$ represents the formation satellite position vector, and $\dot{\boldsymbol{r}}$ is the velocity vector of the formation satellite, and $\hat{\boldsymbol{r}}$ and $\hat{\boldsymbol{r}}$ are the valuations of $\boldsymbol{r}$ and $\dot{\boldsymbol{r}}$. The covariance matrix of the initial process noise is set to:

$$
Q=\operatorname{diag}\left(p_{1}^{2}, p_{1}^{2}, p_{1}^{2}, p_{2}^{2}, p_{2}^{2}, p_{2}^{2}, p_{1}^{2}, p_{1}^{2}, p_{1}^{2}, p_{2}^{2}, p_{2}^{2}, p_{2}^{2}\right)
$$

The noise variance matrix of the pulsar timing observation is set to:

$$
\begin{aligned}
R_{p}= & \operatorname{diag}\left[(0.109 k m)^{2},(0.325 k m)^{2},(0.344 \mathrm{~km})^{2},(0.109 k m)^{2}\right. \\
& \left.,(0.325 k m)^{2},(0.344 \mathrm{~km})^{2}\right]
\end{aligned}
$$

The first three terms show the noise when the reference satellite is used for pulse chronograph observation, and the latter three represent the noise when the satellite is subjected to pulsar timing observation.

Considering the error caused by the inter-satellite baseline measurement, the noise variance matrix of the angle between the pulsar radiation and the inter-satellite baseline is set as:

$$
R_{\alpha}=\left[0.0000009^{2}, 0.0000009^{2}, 0.0000009^{2}\right]
$$

When the reference satellite and satellite with the same track in the run, in accordance with the above parameters of the set method, the binary formation of the situation simulation. In order to compare the accuracy of the state estimation and the accuracy of the satellite state estimation obtained by using the pulsar chronograph, we can only use the pulsed chronograph observation method to realize the autonomous navigation result of the binary formation. In this case, the results of the UKF filter, the result of UKF filtering is shown in Figure VII.

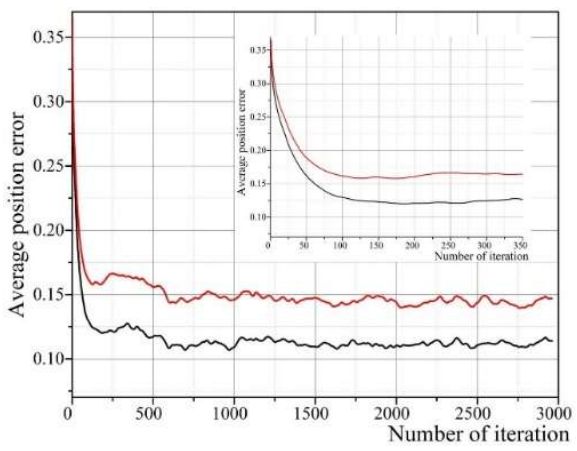



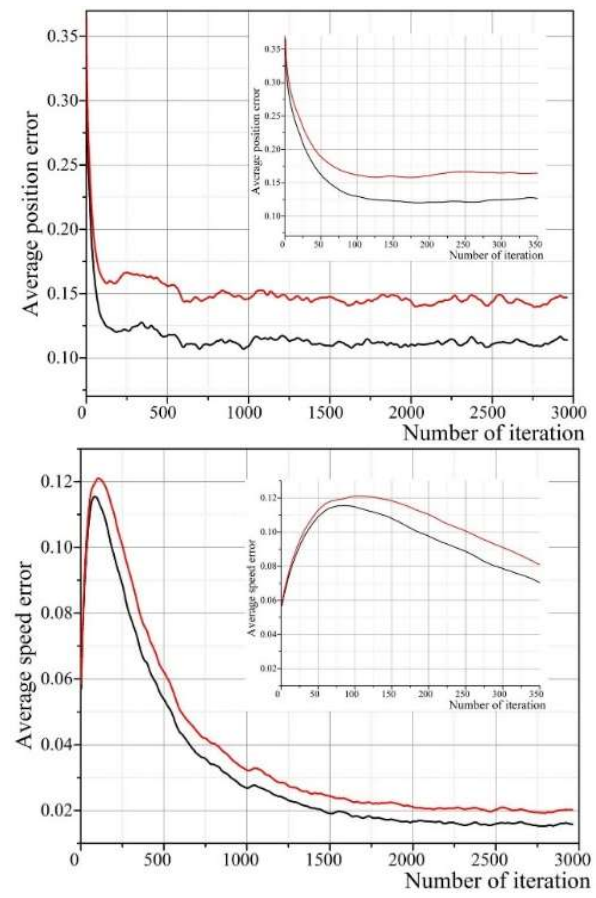

FIGURE VII. COMPARSION OF THIS PAPER METHOD AND TIMING OBVERSION METHOD (SAME ORBITS)

In order to verify the performance of the proposed method and the satellites in different orbital operation, the orbit of the reference satellite is selected as the MEGSAT-1 orbit, and the satellite orbit is selected as the GPS BIIA-10 track, and the other parameters remain unchanged. In this case, use the method given in this paper and the use of pulse timing observation to achieve the formation of satellite autonomous navigation UKF filter comparison chart, as follows:

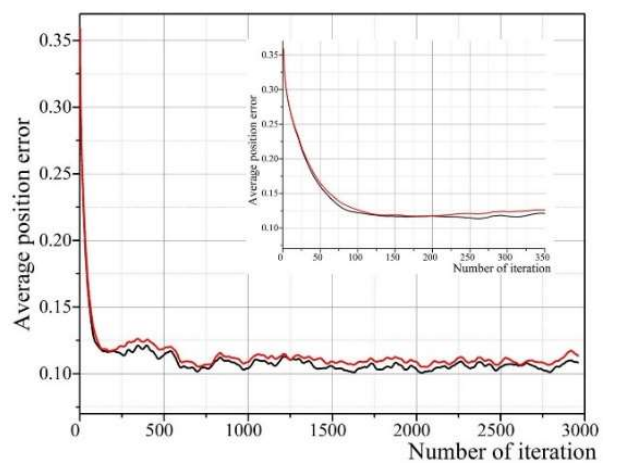

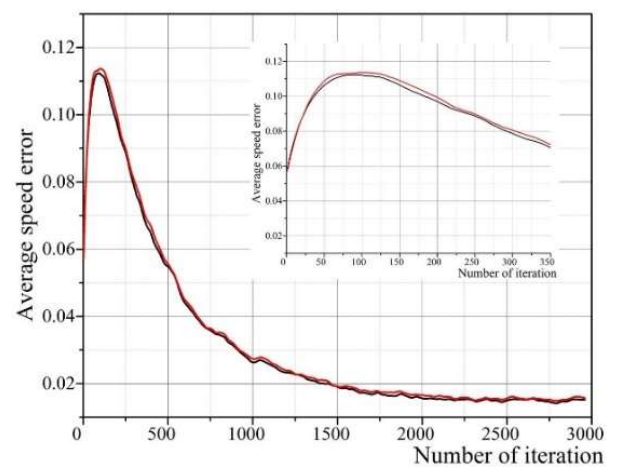

FIGURE VIII. COMPARSION OF THIS PAPER METHOD AND TIMING OBVERSION METHOD (DIFFERENT ORBITS)

Compared with Figure VII and Figure VIII, it can be found that the method presented in this paper has a significant improvement in the convergence rate compared with the pulse timing observation, both in the same orbit and in different orbits, and the position accuracy and velocity accuracy can be improved. Time to obtain a higher accuracy of the satellite status estimates. Therefore, the new method of satellite navigation based on pulsar / baseline information given in this paper is improved in the case of two satellites formation, and the performance is better than that of pulsar time observation method, which has further research value.

\section{Simulation Results of Three Satellites Navigation}

Three X-ray pulsar targets were selected as B0531 + 21, $\mathrm{B} 1937+21$ and B1821-24 when the simulation of a new method of navigation for three satellites formation. The initial error of the formation satellite status is set to:

$$
\delta \mathbf{X}(0)=\left[\begin{array}{l}
\delta \mathbf{X}_{0}(0) \\
\delta \mathbf{X}_{1}(0) \\
\delta \mathbf{X}_{2}(0)
\end{array}\right]
$$

Where $\delta \mathbf{X}_{0}(0)=[0.8 \mathrm{~km} 0.8 \mathrm{~km} 0.8 \mathrm{~km} 5 \mathrm{~m} / \mathrm{s} 5 \mathrm{~m} / \mathrm{s} 5 \mathrm{~m} / \mathrm{s}]^{\mathrm{T}}$ is the position error and velocity at the initial time of the reference satellite. $\delta \mathbf{X}_{1}(0)=[0.8 \mathrm{~km} \quad 0.8 \mathrm{~km} \quad 0.8 \mathrm{~km} 5 \mathrm{~m} / \mathrm{s} \quad 5 \mathrm{~m} / \mathrm{s}$ $5 \mathrm{~m} / \mathrm{s}]^{\mathrm{T}}$ represents the position error and velocity error accompanying the initial time of the satellite A. $\delta \mathbf{X}_{\mathbf{2}}(0)=[0.8 \mathrm{~km} 0.8 \mathrm{~km} 0.8 \mathrm{~km} 5 \mathrm{~m} / \mathrm{s} 5 \mathrm{~m} / \mathrm{s} 5 \mathrm{~m} / \mathrm{s}]^{\mathrm{T}}$ represents the position error and the speed error accompanying the initial time of the satellite B.

The covariance matrix of the initial process noise is set to: $Q=\operatorname{diag}\left(p_{1}^{2}, p_{1}^{2}, p_{1}^{2}, p_{2}^{2}, p_{2}^{2}, p_{2}^{2}, p_{1}^{2}, p_{1}^{2}, p_{1}^{2}, p_{2}^{2}, p_{2}^{2}, p_{2}^{2}, p_{1}^{2}, p_{1}^{2}, p_{1}^{2}, p_{2}^{2}, p_{2}^{2}, p_{2}^{2}\right)$

Where $p_{1}=20 m, \mathrm{p}_{2}=3 \mathrm{~m} / \mathrm{s}$.

The variance matrix of the chronograph observation noise is set to:

$$
R_{p}=\operatorname{diag}\left[(w 1)^{2},(w 2)^{2},(w 3)^{2},(w 1)^{2},(w 2)^{2},(w 3)^{2},(w 1)^{2},(w 2)^{2},(w 3)^{2}\right]
$$

Where $w 1=0.109 \mathrm{~km}, w 2=0.325 \mathrm{~km}, w 3=0.344 \mathrm{~km}$, the first 
three represent the noise when the reference satellite is observed for pulsar chronograph, and the last six represent the observed noise when two satellites are observed with the pulse.

Considering the error caused by the inter-satellite baseline measurement, the noise variance matrix of the angle between the pulsar radiation and the inter-satellite baseline is set as:

$$
R_{\alpha}=\left[0.0000009^{2}, 0.0000009^{2}, 0.0000009^{2}\right]
$$

When the reference satellite and satellite with the same track in the run, in accordance with the above parameters of the set method, the binary formation of the situation simulation. In order to compare the accuracy of the state estimation and the accuracy of the satellite state estimation obtained by using the pulsar chronograph, we can only use the pulsed chronograph observation method to realize the autonomous navigation result of the binary formation. In this case, the results of the UKF filter, the result of UKF filtering is shown in Figure 5.3.
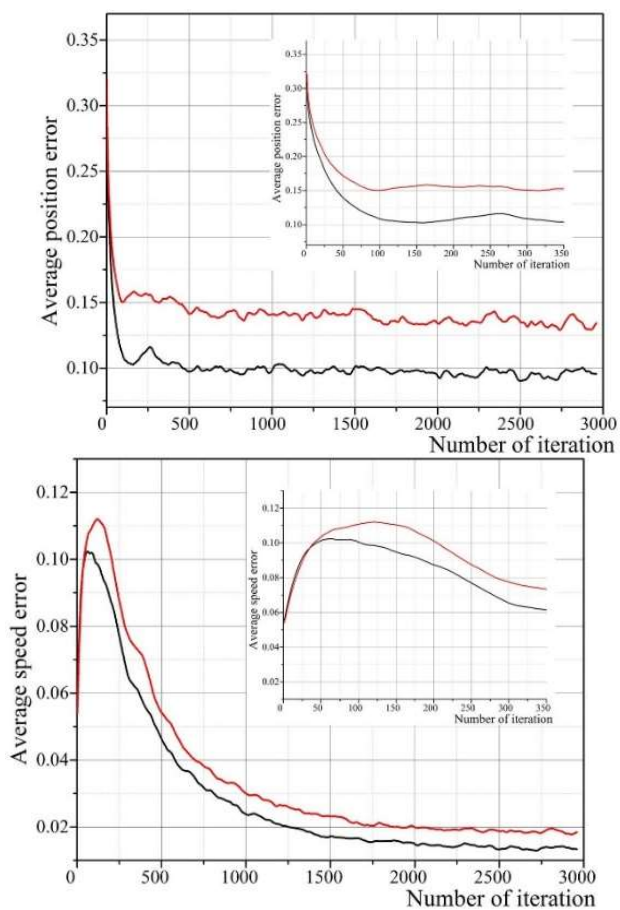

FIGURE IX. COMPARSION OF THIS PAPER METHOD AND TIMING OBVERSION METHOD (SAME ORBITS)

In order to verify the performance of the proposed satellite and the accompanying satellite in different orbits, the orbit of the reference satellite is GPS BIIA-10 orbit, and the orbit accompanied by satellite A and satellite B is GPS BII-3 and GPS BIIR- 4 other parameters remain unchanged. In this case, use the method given in this paper and the use of pulse timing observation to achieve the formation of satellite autonomous navigation UKF filter comparison chart, as follows:
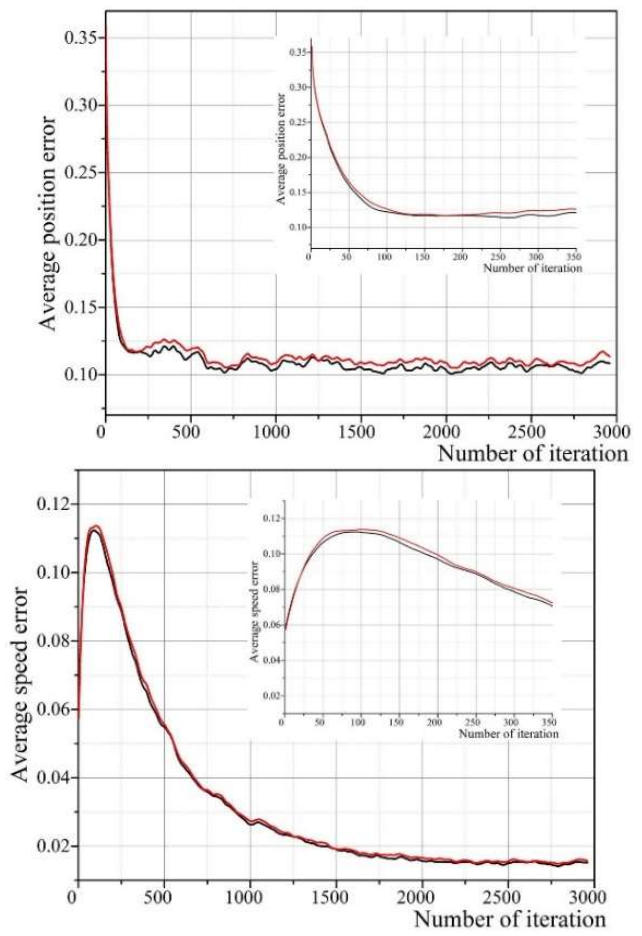

FIGURE X. COMPARSION OF THIS PAPER METHOD AND TIMING OBVERSION METHOD (DIFFERENT ORBITS)

By comparing Fig. IX and Fig. X, it can be seen that in the case of three satellites formation, whether in the same orbit or in different orbits, the new method's convergence rate is faster and the relative error is less than the autonomous navigation method observed by pulsar chronograph. It can be seen that the position estimation error and the velocity estimation error of the three formation satellites are reduced compared with the X-ray pulsar timing observation method, and the position estimation error and the velocity estimation error are reduced by more than $10 \%$. Therefore, the method presented in this paper is feasible to realize the autonomous navigation of the three satellites formation. It can provide a more accurate satellite state estimation in a short time than that of the X-ray pulsar chronograph.

\section{CONCLUSION}

In this paper, the simulation results of the two satellites formation and the simulation results of the three satellites formation are given respectively. It is proved that the method presented in this paper can realize the state estimation of the formation satellites. Compared with the traditional pulsar chronological observation filtering results, the results show that the formation satellite based on pulsar baseline information can better estimate the state of formation satellites.

\section{REFERENCES}

[1] Wang Y, Zheng W, Sun S. X-ray pulsar-based navigation system/Sun measurement integrated navigation method for deep space explorer[J]. Proceedings of the Institution of Mechanical Engineers Part G Journal of Aerospace Engineering, 2015, 229(10):1843-1852.

[2] Yang H X, Vetrisano M, Vasile M, et al. Autonomous navigation of spacecraft formation in the proximity of minor bodies[J]. Proceedings of 
the Institution of Mechanical Engineers Part G Journal of Aerospace Engineering, Vol. 230, Issue 1, pp. 189 - 204, June-24-2015.

[3] Hu Hui-Jun,Zhao Bao-Sheng,Sheng Li-Zhi et al. X-ray photon counting detector for X-ray pulsar-based navigation. Acta Phys. Sin, 2012, 61(1):19701-019701.

[4] Zhang $\mathrm{H}, \mathrm{Xu} \mathrm{L}$ P. Hardware in-the-loop simulation and modeling for XPNAV based on light quantum detection[J]. Journal of Optoelectronics Laser, 2011, 22(6):905-910.

[5] Renga A. A Hybrid Approach for GPS-Based Relative Navigation of Formation Flying Satellites in Remote Sensing Mission[J]. Aidaa, 2016.vol 87,pp.180-190

[6] Park H E, Kim Y R. Relative navigation for autonomous formation flying satellites using the state-dependent Riccati equation filter[J]. Advances in Space Research, 2016, 57(1):166-182.

[7] D'Amico, Simone Montenbruck, Oliver Larsson, Robin Chasset, Camille. (2008). GPS-Based Relative Navigation during the Separation Sequence of the PRISMA Mission. 10.2514/6.2008-6661.

[8] D'Amico S, Montenbruck O, Larsson R, et al. GPS-Based Relative Navigation during the Separation Sequence of the PRISMA Mission[J]. August, 2008

[9] D'Amico S, Gill E, Garcia M F, et al. GPS-Based Real-Time Navigation for the PRISMA Formation Flying Mission[C]// ESA Workshop on Satellite Navigation User Equipment Technologies, Navitec. DLR, 2006:1-8.

[10] Ambrosini M. GPS Precise Relative Positioning of Formation Flying Satellites[J]. Journal of Space Operations \& Communicator, 2016, 14(1).

[11] $\mathrm{Gu} \mathrm{D,} \mathrm{Ju} \mathrm{B,} \mathrm{Liu} \mathrm{J,} \mathrm{et} \mathrm{al.} \mathrm{Enhanced} \mathrm{GPS-based} \mathrm{GRACE} \mathrm{baseline}$ determination by using a new strategy for ambiguity resolution and relative phase center variation corrections[J]. Acta Astronautica, 2017.

[12] Ju B, Gu D, Herring T A, et al. Precise orbit and baseline determination for maneuvering low earth orbiters[J]. Gps Solutions, 2017, 21(1):53-64.

[13] Bentum M J, Verhoeven C J M, Boonstra A J, et al. A novel astronomical application for formation flying small satellites[J]. Nerg Tijdschrift, 2017 76:8-15

[14] Huang L W, Liang B, Zhang T, et al. Navigation using binary pulsars[J]. Science China Physics Mechanics \& Astronomy, 2012, 55(3):527-539.

[15] Xiong Z, Qiao L, Liu J, et al. Geo satellite autonomous navigation using $\mathrm{X}$-ray pulsar navigation and gnss measurements[J]. International Journal of Innovative Computing Information \& Control Ijicic, 2011, 8(5).

[16] Navigation in Space by X-ray Pulsars.Authors: Emadzadeh, Amir Abbas, Speyer, Jason Lee

[17] Liu L, Zheng W, Tang G. Observability analysis of satellite constellations autonomous navigation based on X-ray pulsar measurements[C]// Chinese Automation Congress. IEEE, 2013:148-151.

[18] LIU Yang, YI Dong-yun, WANG Zheng-ming. Research on High Precision Measure Methods of Inter-satellite Baselines of Formation Flying Satellites[J]. Journal of Astronautics , 2007, 28(6) : 1643-1647.

[19] Allende-Alba G, Montenbruck O. Robust and precise baseline determination of distributed spacecraft in LEO[J]. Advances in Space Research, 2016, 57(1):46-63.

[20] Nurge M A, Youngquist R C, Starr S O. A satellite formation flying approach providing both positioning and tracking $[\mathrm{J}]$. Acta Astronautica, 2016, 122:1-9

[21] Mao X, Visser P N A M, Ijssel J A A V D. The application of in-flight antenna pattern corrections to GPS-based baseline determination for formation flying GRACE[C]// International Workshop on Satellite Constellations and Formation Flying. 2015.

[22] LI Jian-xun, KE Xi-zheng. ML Phase Estimate for X-ray Pulsar Signal Based on Nonhomo-geneous Poisson Process[J]. SIGNAL PROCESSING, 2010, 26(8):1252-1256.

[23] Xie Zhen-Hua, Xu Lu-Ping, Ni Guang-Ren. Time offset measurement algorithm based on bispectrum for pulsar integrated pulse profiles[J].ACTA PHYSICA SINICA ,2008,57(10):6683-6688.

[24] Emadzadeh A A, Speyer J L. On modeling and pulse phase estimation of X-ray pulsars[M]. IEEE Press, 2010 , 58 (9) :4484-4495

[25] Emadzadeh A A, Golshan A R, Speyer J L. Consistent estimation of pulse delay for X-ray pulsar based relative navigation[C]// Decision and
Control, 2009 Held Jointly with the 2009. Proceedings of the, IEEE Conference on. IEEE, 2009:1488-1493. 